\title{
Coma Mixedematoso - Relato de caso
}

Bárbara Macedo1; Laura Zaparoli Zanrosso²; Andreza Teixeira Ribeiro ${ }^{3}$ João Augusto Argenton Zortea ${ }^{4}$; Joyce Carvalho Schotten ${ }^{5}$.

\section{RESUMO:}

INTRODUÇÃO: Coma mixedematoso $(\mathrm{CM})$ é uma condição clínica, rara, que se manifesta em pacientes com hipotireoidismo primário ou secundário, de longa duração, e não tratados. Trata-se de emergência metabólica e cardiovascular que, quando não prontamente diagnosticada e tratada, está associada à mortalidade superior a $50 \%$. RELATO DE CASO A.G.S.C, 66 anos, feminino, procurou atendimento em serviço de emergência, com queixa de dor e edema em olho esquerdo há sete dias. Referia dor em queimação e edema periorbitário à esquerda, associado a edema de membros inferiores e confusão mental. Paciente confusa, desacompanhada, referia sono excessivo. Negava fator de alívio ou piora. Sem mais dados relevantes ao caso. Ao exame físico inicial encontrava-se em regular estado geral, mucosas normocoradas, xerose cutânea, edema periorbitário bilateral importante com sinais inflamatórios e secreção mucopurulenta, confusa, desorientada, anictérica, acianótica, afebril, eupneica em ar ambiente. Pressão Arterial: 106/78 mmHg, bradicárdica, frequência respiratória de $18 \mathrm{mrpm}$. Ausculta pulmonar com crepitantes bibasais. Extremidades: frias, edema sem cacifo em membros inferiores. Sem mais alterações. Solicitados exames complementares. Laboratoriais: TSH $76 \mathrm{Um} / \mathrm{L}$, T4 livre 0,08 Um/l; creatinina 1,29. Em revisão de prontuário, evidenciou-se diagnóstico prévio de hipotireoidismo, sem tratamento. Iniciou-se Levotiroxina $100 \mathrm{mcg} / \mathrm{dia}$ e Oxacilina por celulite em face. Paciente evoluiu com melhora dos sintomas e foi encaminhada para acompanhamento em ambulatório de endocrinologia. DISCUSSÃO DO CASO: O caso relatado reflete um exemplo típico de CM em uma paciente com hipotireoidismo não tratado, englobando características clássicas da condição: epidemiologia, sinais, sintomas e achados laboratoriais. Apresentava infecção documentada, identificada como causa da descompensação metabólica, sendo tratada. CONCLUSÃO: Ainda que o CM seja uma condição rara, representa uma emergência na prática clínica, com potencial desfecho fatal. Cabe ao médico reconhecer a sintomatologia e sinais dessas afecções para garantir manejo adequado e assegurar o melhor desfecho

\footnotetext{
1 Médico, residente em Medicina Interna, no Hospital Universitário Mãe de Deus - babsmacedo@hotmail.com

2 Acadêmica do Curso de Medicina da ULBRA (apresentadora) - laura.zanrosso@hotmail.com

3 Acadêmica do Curso de Medicina da ULBRA - andrezatribeiro@gmail.com

${ }^{4}$ Acadêmico do Curso de Medicina da ULBRA - joaozortea@yahoo.com.br

5 Acadêmica do Curso de Medicina da ULBRA - joysch_@hotmail.com
} 
possível, assegurando o acompanhamento para tratamento do hipotireoidismo e outras comorbidades para evitar novos quadros agudos.

\section{Introdução}

Coma mixedematoso (CM) é uma condição clínica, rara, que se manifesta em pacientes com hipotireoidismo primário ou secundário, de longa duração, e não tratados, sendo a manifestação mais grave de hipotireoidismo com uma taxa de mortalidade de $30 \%$ a $60 \%$. É mais comum entre as mulheres mais velhas e quase nunca ocorre em indivíduos com idade inferior a 60 anos de idade.

\section{Relato de Caso}

A.G.S.C, 66 anos, feminino, natural de Criciúma e procedente de Porto Alegre, dona de casa, procurou atendimento em serviço de emergência, com queixa de dor e edema em olho esquerdo há sete dias. Referia dor em queimação e edema periorbitário à esquerda, associado a edema de membros inferiores e confusão mental. Negava fator de alívio ou piora. Paciente confusa, desacompanhada, referia sono excessivo. Negava uso de drogas, alergias, uso contínuo de medicamentos ou comorbidades. Ao exame físico inicial encontravase em regular estado geral, mucosas normocoradas, xerose cutânea, edema periórbitario bilateral importante com sinais inflamatórios e secreção mucopurulenta, confusa, desorientada, anictérica, acianótica, afebril, eupneica em ar ambiente. Pressão Arterial: 106/78 mmHg, frequência cardíaca de 56 bpm, frequência respiratória de $18 \mathrm{mrpm}$. Ausculta cardíaca em ritmo regular, dois tempos, bulhas hipofonéticas, sem sopros; ausculta pulmonar com crepitantes bibasais. Abdome com ruídos hidroaéreos presentes, indolor, sem massas ou visceromegalias palpáveis. Ausência de linfonodos palpáveis; pupilas isofotorreagentes, Escala de coma de Glasgow 15. Extremidades: frias, edema sem cacifo em membros inferiores.

Solicitados exames complementares. Laboratoriais: TSH $76 \mathrm{Um} / \mathrm{L}$, T4 livre 0,08 Um/l, creatinina sérica $1,29 \mathrm{mg} / \mathrm{dL}$; CK-MB 6,2 mg/ml. Demais sem alterações. Em revisão de prontuário, evidenciou-se diagnóstico prévio de hipotireoidismo, sem tratamento. Iniciou-se Levotiroxina $100 \mathrm{mcg} / \mathrm{dia}$ e Oxacilina por celulite em face. Realizado ecografia de tireoide: Tireoide com dimensões reduzidas e ecogenicidade difusamente heterogênea, não se observando nódulos bem definidos. Lobo direito: $1,0 \times 2,79 \times 0,9 \mathrm{~cm}$ - volume 1,2. Lobo esquerdo: 2,1 1 1,1 ×0,8-volume de 1,0. Volume total dos lobos: 2,2 cm. Istmo com espessura 0,3 cm.

Durante internação, paciente evoluiu com melhora no estado geral e de celulite em face. Após avaliação de equipe de endocrinologia, já com paciente com sintomas estabilizados, paciente foi encaminhada para acompanhamento ambulatorial.

\section{Discussão do caso}

O caso relatado reflete um exemplo típico de CM em uma paciente com hipotireoidismo não tratado, englobando características clássicas da condição. A epidemiologia mostra que os casos de CM são mais comuns em mulheres, acima dos 60 anos de idade, assim como no caso descrito.

Sintomas e sinais encontrados, como letargia, confusão mental, edema periorbitário, bradicardia, hipotermia, crepitantes bibasais, extremidades edemaciadas e mal perfundidas, também fazem parte do quadro clássico de CM. Os achados encontrados em exames complementares e que são esperados no CM, incluem: TSH elevado, T4 livre baixo, creatinina sérica elevada, CK-MB elevada, ECG evidenciando bradicardia sinusal e complexos QRS de baixa amplitude; radiografia de tórax, com sinais de congestão pulmonar.

\footnotetext{
1 Médico, residente em Medicina Interna, no Hospital Universitário Mãe de Deus - babsmacedo@hotmail.com

2 Acadêmica do Curso de Medicina da ULBRA (apresentadora) - laura.zanrosso@hotmail.com

3 Acadêmica do Curso de Medicina da ULBRA - andrezatribeiro@gmail.com

${ }^{4}$ Acadêmico do Curso de Medicina da ULBRA - joaozortea@yahoo.com.br

5 Acadêmica do Curso de Medicina da ULBRA - joysch_@hotmail.com
} 
Se houver suspeita clínica de CM, o tratamento deverá ser iniciado imediatamente, mesmo antes da confirmação laboratorial, com medidas de suporte e uso de levotiroxina, bem como, o tratamento da possível causa da descompensação metabólica. É de fundamental importância identificar a causa da descompensação. Frequentemente o evento precipitante do coma mixedematoso é uma infecção oculta ou declarada. No caso descrito, havia a presença de celulite periorbitária. Preditores de mau prognóstico em pacientes com coma mixedema incluem o aumento da idade comprometimento cardiovascular e consciência reduzida.

\section{Conclusão}

Ainda que o CM seja uma condição rara, representa uma emergência na prática clínica, com potencial desfecho fatal. Cabe ao profissional de saúde reconhecer a sintomatologia e sinais dessas afecções para garantir manejo adequado e assegurar o melhor desfecho possível, assegurando o acompanhamento para tratamento do hipotireoidismo e outras comorbidades para evitar novos quadros agudos.

\section{Referências}

BEYNON, J.; AKHTAR, S.; KEARNEY, T. Predictors of outcome in myxoedema coma Endocrinology Department, Salford Royal NHS Foundation Trust, Critical Care 2008, 12:111

MACIEL, LMZ. Coma mixedematoso. Medicina, Ribeirão Preto, 36: 384-388, abr./dez. 2003.

GUPTA, K. Myxedema Coma: A Sleeping Giant in Clinical Practice. The American Journal of Medicine, Vol 126, No 12, 2013.

DUBBS, S.; SPANGLER, R. Hypothyroidism Causes, Killers, and Life-Saving Treatments. Emerg Med Clin N Am 32 (2014) 303-317.

KLUBO-GWIEZDZINSKA, J; WARTOFSY, L. Thyroid Emergencies. Medical Clinics of North America, Thyroid Disorders and Diseases Volume 96, Issue 2, March 2012, Pages 385-403.

RODRIGUEZ I, FLUITER E, PERES-MENDEZ LF, et al. Factors associated with mortality of patients with myxedema coma: prospective study in 11 cases treated in a single institution. J Endocrinol 2004; $80: 347-50$

1 Médico, residente em Medicina Interna, no Hospital Universitário Mãe de Deus - babsmacedo@hotmail.com

2 Acadêmica do Curso de Medicina da ULBRA (apresentadora) - laura.zanrosso@hotmail.com

3 Acadêmica do Curso de Medicina da ULBRA - andrezatribeiro@gmail.com

${ }^{4}$ Acadêmico do Curso de Medicina da ULBRA - joaozortea@yahoo.com.br

5 Acadêmica do Curso de Medicina da ULBRA - joysch_@hotmail.com 